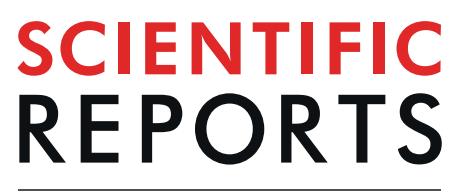

natureresearch

\title{
OPEN New signatures of poor CD4 cell recovery after suppressive antiretroviral therapy in HIV-1- infected individuals: involvement of miR-192, IL-6, sCD14 and miR-144
}

\author{
Francisco Hernández-Walias ${ }^{1,7}$, María J. Ruiz-de-León ${ }^{1,7}$, Isaac Rosado-Sánchez ${ }^{2}$,

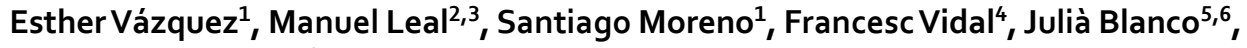 \\ Yolanda M. Pacheco ${ }^{2 *}$ \& Alejandro Vallejo ${ }^{1 *}$
}

Up to $40 \%$ of newly diagnosed cases of HIV-1 infection are late diagnoses, with a profound decrease in CD4 cell counts in many cases. One-third of these individuals do not achieve optimal CD4 cell recovery (OR) after suppressive antiretroviral treatment (ART). This retrospective/longitudinal study of poor recovery (PR) included 79 HIV-1-infected individuals with CD4 count $<200$ cells/mm ${ }^{3}$ ( 25 PR and 54 OR) before ART. After suppressive ART, 21 PR and 24 OR individuals were further analysed, including paired samples. Selected miRs and plasma inflammatory markers were determined to investigate their potential predictive/diagnostic value for poor recovery. miR-192, IL-6 and SCD14 were independently associated with CD4 recovery before ART $(p=0.031, p=0.007$, and $p=0.008$, respectively). The combination of these three factors returned a good discrimination (predictive value for $P R$ ) value of 0.841 (AUC, $p<0.001$ ). After suppressive ART, miR-144 was independently associated with CD4 recovery $(p=0.017)$, showing a moderate discrimination value of 0.730 (AUC, $p=0.008$ ) for PR. Our study provides new evidence on the relationship between miRs and HIV-1 infection that could help improve the management of individuals at HIV-1 diagnosis. These miRs and cytokines signature sets provide novel tools to predict CD4 cell recovery and its progression after ART.

Newly diagnosed cases of HIV-1 infection include individuals with a late diagnosis who often have a low level of CD4 cell counts. Antiretroviral treatment (ART) can restore the CD4 cell level in most of the HIV-1-infected individuals. Nevertheless, one-third of these individuals remained at a very low CD4 level $\left(<200 \mathrm{cells} / \mathrm{mm}^{3}\right)$ after ART despite virological suppression ${ }^{1-3}$.

Persistent immune activation and inflammation are associated with poor CD4 cell recovery (PR). These factors contribute to the risk of illness, increasing the risk of several morbidities compared to the uninfected population, as well as the risk of death ${ }^{4,5}$. While to date no effective alternative treatment is available to increase the CD4 cell levels to optimal counts, initiation of ART early after HIV-1 diagnosis might provide a good opportunity to maximise the $\mathrm{CD} 4$ cell recovery.

On the other hand, adding antiretroviral drugs to an already suppressive treatment does not improve either $\mathrm{CD} 4$ cell recovery nor reduce morbidity or mortality ${ }^{6,7}$. Besides, no observable clinical benefit was observed in

${ }^{1}$ Laboratory of Immunovirology, Department of Infectious Diseases. Health Research Institute Ramon y Cajal (IRyCIS), University Hospital Ramon y Cajal, Madrid, Spain. ${ }^{2}$ Biomedicine Institute of Seville (IBiS), University Hospital Virgen del Rocío, Seville, Spain. ${ }^{3}$ Department of Internal Medicine and Infectious Diseases. Hospital Viamed, Santa Ángela de la Cruz, Seville, Spain. ${ }^{4}$ Infectious Diseases Unit and HIV/AIDS, Department of Internal Medicine, University Hospital Joan XXIII, IISPV, University Rovira i Virgili, Tarragona, Catalonia, Spain. ${ }^{5}$ AIDS Research Institute IrsiCaixa-HIVACAT, Research Institute of Health Sciences Germans Trias i Pujol, Badalona, Catalonia, Spain. ${ }^{6}$ University of Vic-University Central of Catalonia (UVIC-UCC), Vic, Catalonia, Spain. ${ }^{7}$ These authors contributed equally: Francisco Hernández-Walias and María J. Ruiz-de-León. *email: ypacheco-ibis@us.es; alejandro.vallejo@ salud.madrid.org 
IL-2 therapy, although it resulted in CD4 count increases ${ }^{8}$. The use of other immune-based therapies (e.g., growth hormones or IL-7) is controversial and its clinical benefit remains unclear9.

Micro RNAs (miRs) have been largely studied in cancer processes as biomarkers with an immunomodulatory role that might negatively or positively influence the immune system ${ }^{10,11}$. miRs are released inside exosome vesicles by cells and are present in all body fluids investigated to date. Disease presence and progression have been associated with an increase of both exosome release and their molecular content. These molecules could influence the homeostasis cell balance, promoting hematopoietic stem cells and, by modifying the levels of soluble cytokines, regulate the immune system ${ }^{12-14}$. A role for miRs in the pathogenesis of HIV-1 disease has been described ${ }^{15,16}$. The translation of HIV-1 proteins can be repressed by miRs located in resting CD4 cells contributing to the latency of HIV-1. On the other hand, HIV-1 itself can alter the expression of miRs expression influencing the progression of the disease $\mathrm{e}^{17-19}$.

Since exosomes can modulate immune responses and might affect HIV-1 pathogenesis, we conducted this longitudinal study to quantify selected miRs and soluble inflammatory markers in HIV-1-infected individuals at ART onset and after 96 weeks under suppressive ART to investigate their potential predictive and diagnostic value of poor CD4 cell recovery.

\section{Methods}

Study setting and population. This retrospective/longitudinal study of adult HIV-1-infected individuals was performed with samples at ART onset and after 96 weeks of suppressive ART collected from the Spanish AIDS Research Network Cohort (CoRIS) through its HIV Biobank (Spain) ${ }^{20,21}$, and the HIV-1-infected individuals Cohort of the University Hospital Ramon y Cajal (Madrid, Spain). We selected 79 HIV-1-infected individuals with $<200 \mathrm{CD} 4 \mathrm{cells} / \mathrm{mm}^{3}$ at ART onset who matched one of the following situations after 96 weeks under suppressive ART ( $<50 \mathrm{HIV}-1$ RNA copies/mL); (i) those whose CD4 count reached $>250 \mathrm{cells} / \mathrm{mm}^{3}$ with cell increase $>200$ CD 4 cells (OR, Optimal CD4 cell recovery individuals); and (ii) those whose CD4 count did not reach 200 cells $/ \mathrm{mm}^{3}$ with cell increase $<150$ CD 4 cells (PR, Poor CD4 cell recovery individuals). This very restrictive selection criterion allowed the comparison of two groups of individuals with no overlapping in CD4 cell increments that could result in confounding results.

Before ART initiation, 25 individuals with PR and 54 individuals with OR with available plasma samples were included in the study. For comparison, after 96 weeks of suppressive ART (the moment of categorisation into PR and OR), 21 individuals with PR and 24 OR were also analysed. Of them, 15 individuals with PR and 18 individuals with OR had paired samples (samples at ART onset and week 96 after treatment) to analyse the evolution of the biomarkers. Demographic parameters such as age, gender, and route of HIV-1 infection transmission, as well as the time of HIV-1 diagnosis, were collected from all individuals. The Ramón y Cajal Hospital Ethics Committee approved the study, which complied with the stipulations of the Declaration of Helsinki; all individuals gave their written informed consent to participate in this study.

Laboratory measurements. CD4 and CD8 counts were determined in fresh blood with a FACScalibur flow cytometer (Becton Dickinson, Franklin Lanes, NJ, USA). Plasma HIV-1 RNA quantification was measured by quantitative polymerase chain reaction (qPCR, COBAS Ampliprep/COBAS Taqman HIV-1 test, Roche Molecular Systems, Basel, Switzerland) according to the manufacturer's protocol, with a detection limit of $40 \mathrm{HIV}-1$ RNA copies/mL. HCV antibodies were assayed by EIA (Siemens Healthcare Diagnosis, Malvern, Pennsylvania), and plasma HCV RNA quantification by RT-qPCR (COBAS Amplicor, Roche Diagnosis, Barcelona, Spain).

Plasma exosome-derived miR quantification. Briefly, frozen EDTA-plasmas were thawed and sequentially centrifuged to remove cell debris, as described previously ${ }^{22}$. They were then treated with thrombin and DNase to prevent platelet and DNA contamination, respectively, and filtered to eliminate larger vesicles such as large extracellular vesicles and apoptotic bodies. Exosomes were precipitated using the miRCURY Exosome isolation kit (Exiqon A/S, Vedbaek, Denmark), and quantified for exosome content by ExoELISA-ULTRA CD63 assay (SBI System Bioscience, Mountain View, CA, USA).

RNA from isolated exosome-enriched pellet was extracted using miRCURY RNA isolation kit-Biofluids (Exiqon A/S), and UniSp2-4-5 RNA templates added as an internal control. Concentration and purity of eluted RNA were analysed using a NanoDrop instrument (Thermo Scientific) ${ }^{22}$. All RNA samples with 260/280 ratio between 1.8 to 2 and a 260/230 ratio near 2 were considered suitable for further analysis. Ten nanograms of RNA was reverse transcribed in $15 \mu \mathrm{l}$ reactions (mirCURY LNA Universal RT microRNA PCR, Exiqon A/S), including UniSp6 RNA spike-in template reaction control. cDNA was used for PCR reaction in triplicate using ExiLENT SYBR Green Master Mix (Exiqon A/S). LNA-based primers (Exicon A/S) for hsa-miR-451a and hsa-miR-23a were assayed to detect levels of haemolysis. Primers for hsa-miR-103a-3p, hsa-miR-425-5p, and hsa-miR-93-5p were quantified as reference controls for the calculation of the relative concentration of the following miRs: hsa-miR-106a-5p, hsa-miR-140-5p, has-miR-144-5p, hsa-miR-221-3p, hsa-miR-223-3p, hsa-miR-320a-5p, hsa-miR-409-5p, hsa-miR-192-5p, and hsa-miR-24-3p.

Thermocycler conditions were hot start at $95^{\circ} \mathrm{C} / 10 \mathrm{~min}$ followed by 40 cycles of $95^{\circ} \mathrm{C} / 15 \mathrm{~s}$ and $60^{\circ} \mathrm{C} / 45 \mathrm{~s}$ using LyghtCycler 480 II instrument (Roche, Basel, Switzerland). Amplification curves were analysed by Roche LightCycler 480 version 1.5.1.62 software. Reaction specificity was ascertained by performing the melt curve procedure. Ct values $>35$ and a standard deviation between triplicates $>0.3 \mathrm{Ct}$, were considered unreliable and excluded from further analysis. The expression levels of single miRs relative to the mean reference miRs expression were calculated using the $\Delta \mathrm{Ct}$ method $(\mathrm{Ct} \text { target miR minus mean references } \mathrm{Ct})^{23-25}$ and reported as $\log _{2} 2^{-\Delta \mathrm{Ct}}$. 
Soluble plasma biomarkers. Plasma samples were used to quantify the following markers of inflammation and immune activation: interleukin-6 (IL-6), soluble CD14 (sCD14), tumour necrosis factor-alpha (TNF $\alpha$ ), IL-2, IL-17A, ICAM, and VCAM, using R\&D Luminex HS assay (R\&D Systems MN, USA). Human Procarta-Plex immunoassays (ThermoFisher Scientific) were used in combination with the Luminex instrument platform (MagPix, Luminex Corporation), following the manufacturer's instructions.

Statistical analysis. Continuous variables were expressed as the median and interquartile range $\left(\mathrm{IQ}_{25-75}\right)$, and categorical variables by frequencies and proportions. The Mann-Whitney U test (non-parametric) for independent samples was used to compare continuous variables. Differences between categorical variables were evaluated using a contingency table (Chi-square distribution). For the categorical dependent variable (PR vs OR), univariate logistic regression analysis was assessed using all variables studied. Variables with $\mathrm{p}<0.1$ in the univariate analysis were included in the multivariate logistic regression analysis with a stepwise enter method. The regression coefficient $(\beta)$ and $95 \%$ confidence interval for $\beta$ were estimated in this model. Variables with $p<0.05$ were independently associated with the dependent variable. The Wilcoxon signed-rank test was used to compare pared-samples to analyse the evolution of the biomarkers. Spearman's rank correlation coefficient was used to measure the association between two variables. Besides, receiver operating characteristic (ROC) and area under the curve (AUC) were used to evaluate the diagnostic potential (with 95\% confidence intervals), and to calculate the sensitivity and specificity of the biomarkers in CD4 cell recovery. Also, the likelihood ratio was performed to assess how these variables increase or decrease the probability to have poor CD4 recovery. Statistical analysis was performed using SPSS software 22.0 (SPSS Inc., Chicago, Illinois, USA).

\section{Results}

The immunovirologic characteristics of the individuals at ART onset, classified subsequently as having either PR or OR, are shown in Table 1 . The mean age of individuals was 40 years and were mainly males in both groups. No differences were found on the route of HIV-1 transmission, time from HIV-1 diagnosis to ART initiation, HIV-1 RNA load, and rates of HCV infection between the two groups of individuals. CD4 counts were lower in PR individuals, although this did not reach statistical significance $(\mathrm{p}=0.076)$, while CD8 counts were similar in both groups. Nevertheless, the CD4/CD8 ratio was significantly lower in individuals with $P R(p=0.022)$.

The type of antiretroviral treatment prescribed was similar in both groups $(\mathrm{p}=0.252)$, as shown in Table 1 . Most of the PR individuals (56\%) received two nucleoside/nucleotide reverse transcriptase inhibitors (NRTIs) plus one protease inhibitor (PI). Emtricitabine plus tenofovir were the most prescribed NRTI combination (47.8\%), while the most prescribed PIs were ABT (38.8\%) and atazanavir potentiated with ritonavir (17.4\%). Nine PR individuals (36\%) received two NRTIs plus one non-nucleoside reverse transcriptase inhibitor (NNRTI), with emtricitabine plus tenofovir as the most prescribed combination of NRTIs (80.7\%), and efavirenz as the most prescribed NNRTI (92.3\%). Among the OP individuals who received two NRTIs plus one PI (42.6\%), emtricitabine plus tenofovir was again the most prescribed combination of NRTI (78.6\%), while either lopinavir, ABT, or darunavir englobed the $78.5 \%$ of the PIs. Twenty-six OP individuals (48.1\%) received two NRTIs plus one NNRTI, with emtricitabine plus tenofovir as the most prescribed combination of NRTIs (55.6\%), and either nevirapine (44.4\%) or efavirenz $(55.6 \%)$ as the prescribed NNRTI.

After 96 weeks under suppressive ART (mostly composed by two nucleoside analogue inhibitor plus one protease inhibitor), CD4 counts, CD8 counts, and CD4/CD8 ratio were significantly lower in individuals with PR $(\mathrm{p}<0.001, \mathrm{p}=0.023$, and $\mathrm{p}=0.001$, respectively) (Table 1$)$. Also, the CD4 cell increase was lower in individuals with $\mathrm{PR}(\mathrm{p}<0.001)$ as a consequence of the group definition criteria.

Exosome-derived miRs and soluble cytokines with predictive value for CD4 cell recovery at ART onset. miR-106a and miR-140 levels were lower in individuals with $P R(p=0.001$ and $p=0.002$, respectively), while the miR-192 level was higher $(\mathrm{p}=0.001)$ compared to individuals with OR (Table 2A). Besides, similar levels of the rest of the miRs were found in the two groups of individuals. Plasma IL-2 levels were lower in individuals with PR compared to individuals with OR $(\mathrm{p}=0.017)$, while the levels of IL- 6 and sCD14 were higher $(\mathrm{p}<0.001$ and $\mathrm{p}=0.046$, respectively) (Table $2 \mathrm{~B}$ ). Also, individuals with PR showed higher VCAM and ICAM levels compared to those with OR, although not reaching the limit of statistical significance $(p=0.051$ and $p=0.055$, respectively). The levels of the rest of the soluble biomarkers were similar between the two groups of individuals.

To investigate whether gender or HCV infection could have had any effect on the levels of the variables studied, only individuals with OR were analysed since the number of individuals with PR (only four females and three $\mathrm{HCV}$-infected individuals) was not enough to give good statistical power in the comparison. Hence, among individuals with OR no statistical differences in the levels of the miRs and cytokines were found between females $(n=8)$ and males $(\mathrm{n}=46)$ (Supplementary Tables S1A,B). Regardless that the diagnosis of HCV infection was not documented in a large number of individuals, an analysis of the impact of HCV infection among individuals with OR was performed including 12 individuals with OR with positive anti-HCV antibodies and positive HCV PCR, and 20 individuals with OR with negative anti-HCV antibodies. Individuals with unknown HCV PCR were not included in the analysis because there could be cases of spontaneous HCV clearance that would interfere with the results. Hence, no significant differences were found in the levels of miRs between individuals with or without HCV infection, except for miR-192 that was shown to be higher in individuals with PR ( $p=0.010)$ (Supplementary Table S1A).

Exosome-derived miR-192, IL-6, and SCD14 were independently associated with CD4 cell recovery at ART onset. To identify which biomarkers were independently associated with CD4 cell recovery, multivariate logistic regression analysis ( $P R$ vs $\mathrm{OR}$ ) was performed including all variables with $\mathrm{p}<0.1$ in the univariate logistic regression test (Table 3 ). Hence, miR-192, IL-6, and sCD14 were independently associated to $\mathrm{CD} 4$ cell recovery $(\mathrm{p}=0.031, \mathrm{p}=0.007$, and $\mathrm{p}=0.008$, respectively). In addition, while no correlation between 


\begin{tabular}{|c|c|c|c|}
\hline & $\begin{array}{l}\text { Poor CD4 T cell } \\
\text { recovery individuals }\end{array}$ & $\begin{array}{l}\text { Optimal CD4 T cell } \\
\text { recovery individuals }\end{array}$ & $\mathbf{p}$ \\
\hline $\mathrm{N}$ & 25 & 54 & \\
\hline Age (years) & $41[35-52]$ & 39 [34-48] & 0.340 \\
\hline Gender (males, \%) & $21(84 \%)$ & $46(85.2 \%)$ & 0.875 \\
\hline Route of HIV-1 transmission* & & & 0.287 \\
\hline Injecting drug use $(\mathrm{N}, \%)$ & $4(16 \%)$ & $10(18.5 \%)$ & \\
\hline Sexual intercourse $(\mathrm{N}, \%)$ & $16(64 \%)$ & $38(70.4 \%)$ & \\
\hline Unknown & $5(20 \%)$ & $6(11.1 \%)$ & \\
\hline HIV-1 diagnosis to ART initiation (months) & $1.0[0.2-2.0]$ & $2.0[1.0-26.0)$ & 0.147 \\
\hline Type of ART initiated* & & & 0.252 \\
\hline 2 NRTIs + 1 PI & $14(56 \%)$ & $23(42.6 \%)$ & \\
\hline 2 NRTIs + 1 NNRTI & $9(36 \%)$ & $26(48.1 \%)$ & \\
\hline 2 NRTIs + 1 INSTI & $1(4 \%)$ & $3(5.6 \%)$ & \\
\hline 3 NRTIs & $1(4 \%)$ & $2(3.7 \%)$ & \\
\hline HIV-1 RNA load ( $\log _{10}$ copies/mL) & $5.01[4.23-5.33]$ & $5.10[4.69-5.51]$ & 0.197 \\
\hline HCV infection* & & & 0.349 \\
\hline Positive for anti-HCV antibodies & $5(20 \%)$ & $14(25.9 \%)$ & \\
\hline HCV PCR positive & 3 & 12 & \\
\hline HCV PCR unknown & 2 & 2 & \\
\hline Negative for anti-HCV antibodies & $8(32 \%)$ & $20(37 \%)$ & \\
\hline Unknown for anti-HCV antibodies & $12(48 \%)$ & $20(37 \%)$ & \\
\hline CD4 T cell count $\left(\right.$ cells $\left./ \mathrm{mm}^{3}\right)$ & $84[57-146]$ & $139[67-171]$ & 0.076 \\
\hline CD8 T cell count $\left(\right.$ cells $\left./ \mathrm{mm}^{3}\right)$ & $645[464-831]$ & 678 [434-912] & 0.804 \\
\hline CD4/CD8 ratio & $0.11[0.07-0.23]$ & $0.20[0.14-0.27]$ & 0.022 \\
\hline \multicolumn{4}{|l|}{96 weeks after cART onset } \\
\hline CD4 T cell count $\left(\right.$ cells $\left./ \mathrm{mm}^{3}\right)$ & $192[136-214]$ & 429 [362-563] & $<0.001$ \\
\hline CD8 T cell count $\left(\right.$ cells $\left./ \mathrm{mm}^{3}\right)$ & 553 [388-1066] & 867 [692-1109] & 0.023 \\
\hline $\mathrm{CD} 4 / \mathrm{CD} 8$ ratio & $0.26[0.17-0.46]$ & $0.53[0.39-0.70]$ & 0.001 \\
\hline CD4 T cell increment & $77[54-124]$ & $318[248-437]$ & $<0.001$ \\
\hline
\end{tabular}

Table 1. Immunovirologic characteristics of the patients at cART onset (predictive time point) and 96 weeks after cART (diagnosis time point). Median and interquartile range (IQ25-75) for continuous variables, and frequencies and proportions for categorical values. ART, antiretroviral treatment. NRTI,nucleoside/nucleotide reverse transcriptase inhibitor; NNRTI, non-nucleoside reverse transcriptase inhibitor; PI, protese inhibitor; INSTI, integrase strand transfer inhibitor, Mann Whitney $U$ test for continuous values and * Fisher's exact test (two tailed) for categorical values. Significant when $\mathrm{p}<0.05$ in bold.

these three biomarkers was found, miR-192 correlated directly with TNF- $\alpha(\mathrm{r}=0.524, \mathrm{p}=0.014)$ and ICAM $(\mathrm{r}=0.536, \mathrm{p}=0.010)$, and inversely with IL17A $(\mathrm{r}=-0.458, \mathrm{p}=0.063)$. Besides, miR-106a correlated inversely with IL-6 $(r=0.479, p=0.041)$, while miR-140 correlated directly with IL-2 $(r=0.477, p=0.45)$ and inversely with $\mathrm{sCD} 14(\mathrm{r}=0.481, \mathrm{p}=0.039)$.

ROC curves of miR-192, IL- 6 and sCD14 showed moderate discrimination with an AUC value of 0.725 (95\% CI: 0.605-0.845), 0.760 (95\% CI: 0.650-0.870), and 0.640 (95\% CI: 0.514-0.767), respectively (Fig. 1). Nevertheless, the combination of these three factors showed stronger discrimination with an AUC value of 0.841 (95\% CI 0.752-0.929). The sensitivity and specificity of miR-192, IL-6, sCD14, and the combination of these three factors to predict PR with an optimal cut-off value are summarised in Supplementary Table S2. The combination of these factors increases by 3.16-times the probability to predict poor CD4 cell recovery (likelihood ratio analysis). No correlation between miR-192 and CD4 count was found (data not shown), while IL-6 and sCD14 negatively correlated with CD4 count $(\mathrm{p}=0.004$ and $\mathrm{p}<0.001$, respectively).

Levels of exosome-derived miRs and soluble cytokines after 96 weeks of suppressive ART. Twenty-one individuals with PR (15 individuals with samples at ART onset) and 24 individuals with OR (18 individuals with samples at ART onset) were analysed after 96 weeks of suppressive ART (Supplementary Table S3). As expected, the CD4 count and CD4/CD8 ratio were lower in individuals with $\mathrm{PR}$ ( $\mathrm{p}<0.001$ and $\mathrm{p}=0.002$, respectively), while the CD8 count was similar in both groups.

After suppressive ART, miR-106a, miR-140, and miR-144 showed lower levels in individuals with PR $(\mathrm{p}=0.003, \mathrm{p}=0.048$ and $\mathrm{p}=0.008$, respectively) (Table 4A). IL-2 levels were lower in individuals with $\mathrm{PR}$ $(\mathrm{p}=0.014)$, while the levels of IL- 6 and sCD14 were higher in those individuals $(\mathrm{p}=0.005$ and $\mathrm{p}=0.002$, respectively) (Table 4B). 


\begin{tabular}{|l|l|l|l|}
\hline \multicolumn{5}{|l|}{} & $\begin{array}{l}\text { Poor CD4 T cell recovery } \\
\text { patients N=25 }\end{array}$ & $\begin{array}{l}\text { Optimal CD4 T cell } \\
\text { recovery patients N=54 }\end{array}$ & p \\
\hline \multicolumn{5}{|l|}{ A. miRs } & $-1.36[-2.52--0.27]$ & $0.09[-0.98-1.36]$ & $\mathbf{0 . 0 0 1}$ \\
\hline miR-106a & $-1.47[-2.54--0.35]$ & $-0.36[-1.26-0.64]$ & $\mathbf{0 . 0 0 2}$ \\
\hline miR-140 & $-1.79[-4.18--0.37]$ & $-3.41[-4.62--1.26]$ & 0.219 \\
\hline miR-144 & $-1.08[-2.19-0.16]$ & $-0.75[-1.61-0.40]$ & 0.141 \\
\hline miR-221 & $2.87[1.59-4.24]$ & $2.97[1.39-4.89]$ & 0.891 \\
\hline miR-223 & $1.66[0.72-2.54]$ & $1.63[0.53-2.92]$ & 0.929 \\
\hline miR-320a & $-2.96[-4.18--0.49]$ & $-3.69[-4.66--0.49]$ & 0.212 \\
\hline miR-409 & $0.70[-0.91-2.18]$ & $-1.24[-1.74-0.03]$ & $\mathbf{0 . 0 0 1}$ \\
\hline miR-192 & $2.04[0.38-3.02]$ & $1.84[0.13-3.57]$ & 0.887 \\
\hline miR-24 & \multicolumn{5}{|l|}{} \\
\hline B. Cytokines & $0.71[0.57-0.99]$ & $0.98[0.64-1.35]$ & $\mathbf{0 . 0 1 7}$ \\
\hline IL-2 $(\mathrm{pg} / \mathrm{ml})$ & $8.29[5.53-10.95]$ & $4.52[2.89-6.49]$ & $<\mathbf{0 . 0 0 1}$ \\
\hline IL-6 $(\mathrm{ng} / \mathrm{mL})$ & $0.69[0.45-0.99]$ & $0.77[0.59-1.06]$ & 0.244 \\
\hline IL-17A $(\mathrm{pg} / \mathrm{mL})$ & $4.12[2.44-5.32]$ & $2.55[1.45-4.33]$ & 0.056 \\
\hline TNF- $\alpha(\mathrm{pg} / \mathrm{mL})$ & $3.19[2.51-4.01]$ & $2.89[1.76-3.40]$ & $\mathbf{0 . 0 4 6}$ \\
\hline sCD14 $(\mathrm{ng} / \mathrm{ml})$ & $3.74[3.01-4.76]$ & $3.38[2.44-4.14]$ & 0.051 \\
\hline ICAM $\left(\log { }_{10} \mathrm{ng} / \mathrm{mL}\right)$ & $1.12[0.80-1.45]$ & $0.90[0.62-1.22]$ & 0.055 \\
\hline VCAM $\left(\log { }_{10} \mathrm{ng} / \mathrm{mL}\right)$ & & & \\
\hline & &
\end{tabular}

Table 2. miRNAs and cytokines levels of the patients at cART onset. Median and interquartile range (IQ25-75). Mann Whitney U test. Significant when $\mathrm{p}<0.05$ in bold.

\begin{tabular}{|c|c|c|}
\hline & \multicolumn{2}{|l|}{ CD4 $\mathrm{T}$ cell recovery } \\
\hline & $\begin{array}{l}\text { Univariate logistic regression } \\
\mathrm{p}, \beta \text { coefficient }(95 \% \mathrm{CI})\end{array}$ & $\begin{array}{l}\text { Multivariate logistic regression } \\
\text { p, } \beta \text { coefficient }(95 \% \text { CI })\end{array}$ \\
\hline CD4 $\mathrm{T}$ cell count & $0.083,0.993(0.985-1.001)$ & \\
\hline CD4/CD8 ratio & $0.035,0.001(0.000-0.589)$ & \\
\hline miR-106a & $0.001,0.568(0.402-0.802)$ & \\
\hline $\mathrm{miR}-140$ & $0.001,0.465(0.292-0.741)$ & \\
\hline miR-221 & $0.096,0.755(0.542-1.052)$ & \\
\hline miR-192 & $0.002,1.609(1.198-2.162)$ & $\mathbf{0 . 0 3 1}, 1.725(1.052-2.830)$ \\
\hline IL-2 & $0.015,0.178(0.045-0.712)$ & \\
\hline IL-6 & $0.001,1.290(1.104-1.507)$ & $\mathbf{0 . 0 0 7}, 1.497(1.114-2.011)$ \\
\hline sCD14 & $0.003,2.413(1.345-4.330)$ & $\mathbf{0 . 0 0 8}, 5.276(1.554-17.915)$ \\
\hline ICAM & $0.063,1.479(0.980-2.234)$ & \\
\hline VCAM & $0.085,2.769(0.867-8.838)$ & \\
\hline
\end{tabular}

Table 3. Uni and multivariate analysis to investigate which variable was independently associated with CD4 $\mathrm{T}$ cell recovery (dependent variable poor vs. optimal CD4 T cell recovery). Only variables with $\mathrm{p}<0.1$ in the univariate logistic regression were analysed in the multivariate logistic regression analysis. CI: confidence interval. Significant when $\mathrm{p}<0.05$.

Exosome-derived miR-144 was independently associated with CD4 cell recovery after 96 weeks of suppressive ART. After performing multivariate logistic regression analysis with all variables with $\mathrm{p}<0.1$ in the univariate logistic regression test, only miR-144 was independently associated with CD4 cell recovery $(\mathrm{p}=0.017)$ after 96 weeks of suppressive ART (Table 5). Also, the ROC curve of miR-144 showed moderate discrimination with an AUC value of 0.730 (95\% CI: 0.579-0.882) (Fig. 1). The sensitivity and specificity of miR-144 to diagnose PR with an optimal cut-off value are summarised in Supplementary Table S2. This miR increased by 2.16-times the probability to diagnose poor CD4 recovery. Of note, miR-144 positively correlated with CD4/CD8 ratio $(\mathrm{p}=0.006)$ and almost correlated with CD4 count $(\mathrm{p}=0.082)$.

Evolution of the levels of exosome-derived miRs and soluble cytokines after 96 weeks of suppressive ART. Individuals with paired samples were analysed to investigate the evolution of the levels of the biomarkers between these two groups during suppressive ART. Hence, the levels of miR-106a, miR-140, miR-144, and miR-409 significantly increased only in individuals with $\mathrm{OR}(\mathrm{p}=0.001, \mathrm{p}=0.014, \mathrm{p}=0.008$, and $\mathrm{p}=0.006$, respectively), while similar levels of these miRs were found in individuals with PR (Fig. 2). In contrast, the levels of miR-320a and miR-192 decreased significantly only in individuals with $P R(p=0.012$ and $p=0.002$, respectively). Also, miR-24 significantly decreased in both groups of individuals ( $p=0.004$ and $p=0.012$, respectively). 
miR-192

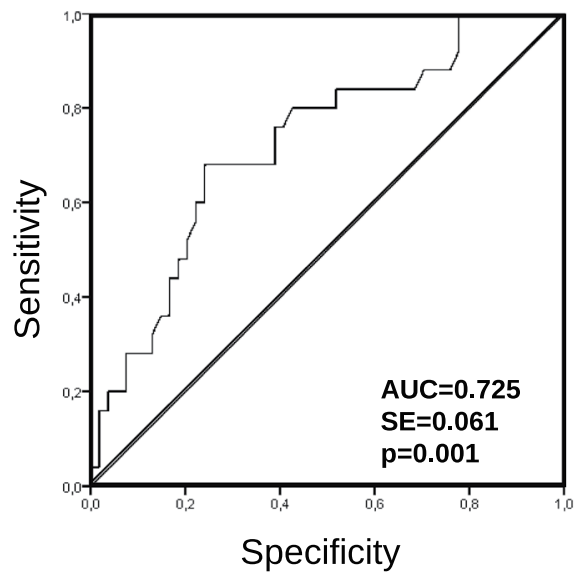

sCD14

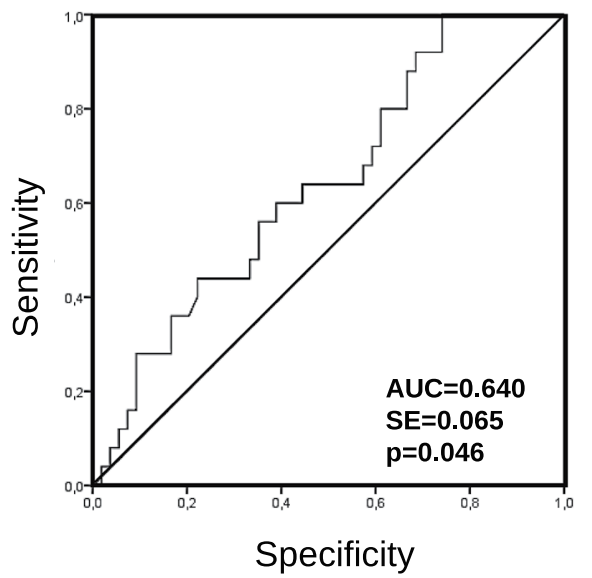

IL-6

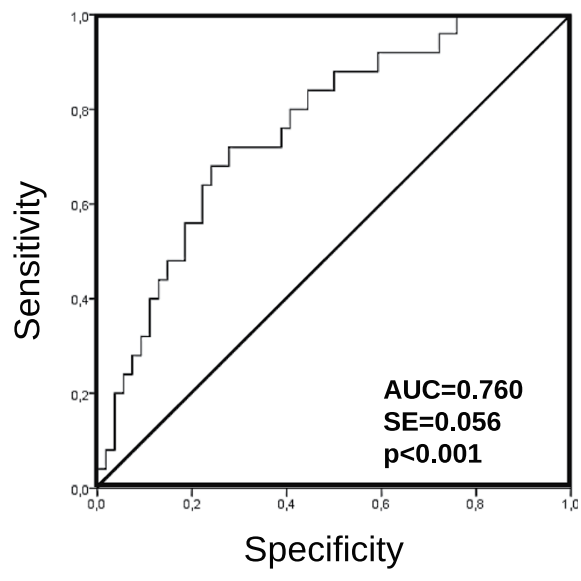

miR-192-IL-6-sCD14 combined

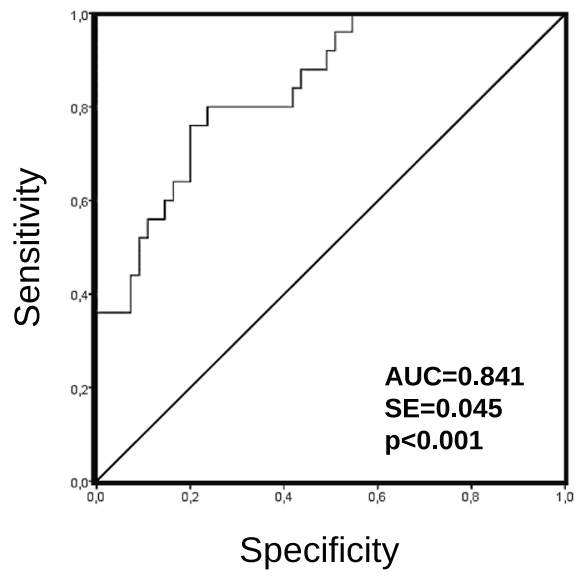

miR-144

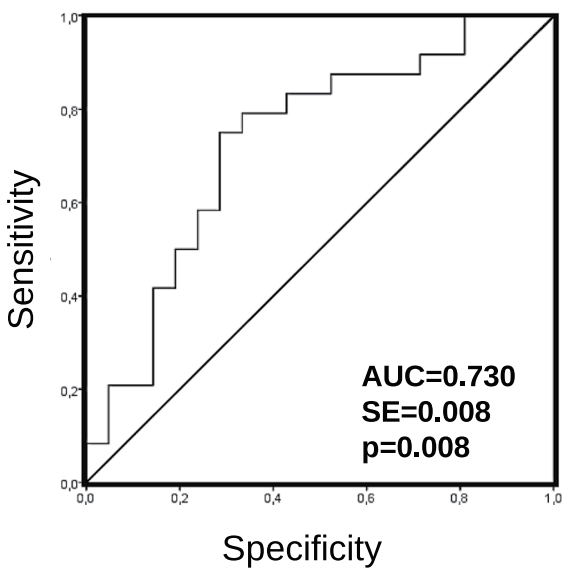

Figure 1. Receive operating characteristic (ROC) curves of differentially expressed miR-192, IL-6, sCD14 and the combination of these three factors between individuals with poor and optimal CD4 T cell recovery at ART onset, and miR-144 after 96 weeks of suppressive ART. AUC, area under the curve; SE, standard error.

The levels of IL- 2 and TNF- $\alpha$ significantly decreased in individuals with OR ( $p=0.010$ and $p=0.011$, respectively), while the level of VCAM decreased only in individuals with PR $(\mathrm{p}=0.002)$. On the other hand, the levels of IL-6, sCD14, and ICAM significantly decreased in both groups of individuals, while the level of IL17A significantly increased in both groups of individuals. Both miR-106a and miR-140 correlated directly with IL2 $(\mathrm{r}=0.586, \mathrm{p}=0.021$, and $\mathrm{r}=0.595, \mathrm{p}=0.017$, respectively), while miR-192 correlated inversely with IL17A $(\mathrm{r}=0.586, \mathrm{p}=0.021)$. No correlation of miR-144 with inflammation markers was found. 


\begin{tabular}{|c|c|c|c|}
\hline & $\begin{array}{l}\text { Poor CD4 T cell } \\
\text { recovery patients } \\
\mathrm{N}=21\end{array}$ & $\begin{array}{l}\text { Optimal CD4T cell } \\
\text { recovery patients } \\
\mathrm{N}=\mathbf{2 4}\end{array}$ & p \\
\hline \multicolumn{4}{|l|}{ A. miRs } \\
\hline miR-106a & $-0.25[-5.31-2.28]$ & $2.74[0.81-3.60]$ & 0.003 \\
\hline miR-140 & $0.89[-5.30-1.75]$ & $1.71[-2.52-3.00]$ & 0.048 \\
\hline miR-144 & $-3.93[-5.22-2.46]$ & $-2.44[-2.93-1.50]$ & 0.008 \\
\hline miR-221 & $-1.34[-4.10-0.61]$ & $-0.78[-2.46-0.31]$ & 0.045 \\
\hline miR-223 & $3.49[2.25-4.39]$ & $3.81[2.79-4.62]$ & 0.317 \\
\hline miR-320a & $1.72[0.23-2.32]$ & $1.74[1.00-2.42]$ & 0.601 \\
\hline miR-409 & $-1.25[-3.17-0.45]$ & $-1.32[-2.01-0.19]$ & 0.873 \\
\hline miR-192 & $-0.89[-1.87-0.19]$ & $-1.20[-2.12-0.08]$ & 0.577 \\
\hline miR-24 & $-0.30[-1.26-1.92]$ & $0.35[-1.00-1.53]$ & 0.419 \\
\hline \multicolumn{4}{|l|}{ B. Cytokines } \\
\hline IL-2 (pg/ml) & $0.61[0.41-0.85]$ & $0.97[0.69-1.14]$ & 0.014 \\
\hline IL-6 (ng/mL) & $3.82[3.27-5.16]$ & $2.41[1.37-4.27]$ & 0.005 \\
\hline IL-17A (pg/mL) & $1.29[0.92-1.54]$ & $1.48[1.17-1.83]$ & 0.052 \\
\hline TNF- $\alpha(\mathrm{pg} / \mathrm{mL})$ & $2.64[1.61-4.13]$ & $1.97[1.24-2.79]$ & 0.162 \\
\hline $\mathrm{sCD} 14(\mu \mathrm{g} / \mathrm{ml})$ & $2.44[2.12-2.94]$ & $1.89[1.51-2.36]$ & 0.002 \\
\hline ICAM $\left(\log _{10} \mathrm{ng} / \mathrm{mL}\right)$ & $2.05[1.53-2.92]$ & $1.66[1.34-2.31]$ & 0.069 \\
\hline VCAM $\left(\log _{10} \mathrm{ng} / \mathrm{mL}\right)$ & $0.79[0.58-0.96]$ & $0.59[0.50-0.87]$ & 0.097 \\
\hline
\end{tabular}

Table 4. Post suppressive cART levels (at week 96) of miRs and cytokines of the patients. Median and interquartile range (IQ25-75). Mann Whitney U test. Significant when $\mathrm{p}<0.05$ in bold.

\begin{tabular}{|l|l|l|}
\hline \multirow{2}{*}{} & \multicolumn{2}{|l|}{ CD4 T cell recovery (poor vs. optimal recovery) } \\
\cline { 2 - 3 } & $\begin{array}{l}\text { Univariate logistic regression } \\
\mathbf{P}, \beta \text { coefficient }(\mathbf{9 5} \% \mathbf{C I})\end{array}$ & $\begin{array}{l}\text { Multivariate logistic regression } \\
\mathbf{p}, \beta \text { coefficient } \mathbf{9 5 \%} \text { CI) }\end{array}$ \\
\hline CD4/CD8 ratio & $0.023 ; 0.000(0.000-0.277)$ & \\
\hline miR-106a & $0.003 ; 0.688(0.538-0.881)$ & \\
\hline miR-140 & $0.098 ; 0.852(0.704-1.030)$ & \\
\hline miR-144 & $0.017 ; 0.618(0.417-0.916)$ & $\mathbf{0 . 0 1 7}, 0.089(0.012-0.654)$ \\
\hline IL-2 & $0.039 ; 0.680(0.471-0.981)$ & \\
\hline IL-6 & $0.027 ; 0.114(0.017-0.785)$ & \\
\hline IL-17A & $0.010 ; 1.769(1.145-2.734)$ & \\
\hline sCD14 & $0.053 ; 0.201(0.040-1.019)$ & \\
\hline ICAM & $0.006 ; 5.564(1.639-18.884)$ & \\
\hline
\end{tabular}

Table 5. Uni and multivariate analysis to assess which variable was independently associated with CD4 T cell recovery (dependent variable poor vs. optimal CD4 T cell recovery) after 96 weeks of suppressive cART. Only variables with $\mathrm{p}<0.1$ in the univariate logistic regression were analysed in the multivariate logistic regression analysis. CI: confidence interval. Significant when $\mathrm{p}<0.05$.

\section{Discussion}

To find predictive biomarkers at ART onset to identify earlier those HIV-1-infected individuals who will have poor CD4 cell recovery after suppressive ART is crucial for the good management of HIV-1-infected individuals $^{4,26}$. This study showed that, at ART onset, lower expression of miR-106a and miR-140, and higher expression of miR-192 could be found in individuals who, thereafter, will be classified as having poor CD4 cell recovery. In parallel, higher levels of IL-6 and sCD14 and lower levels of IL- 2 were found in these individuals. These results provide these biomarkers with a remarkable value that deserves to be further analysed. Interestingly, multivariate analysis showed that miR-192, IL-6, and sCD14 biomarkers were independently associated with CD4 cell recovery at ART onset, and could help strongly identify individuals with subsequent poor CD4 cell recovery after suppressive ART. These differences were independent of both gender and HCV infection (tested among individuals with optimal CD4 cell recovery) except for miR-192, whose level was significantly lower in individuals with HCV infection. This shows that HCV infection could modify the expression of some miRs. Also, the combination of miR-192, IL-6, and sCD14 provided good predictive value with a slight increase (by 3.16-times) in the probability to predict poor CD4 cell recovery.

Little is known regarding miRs profiles in HIV-1-infected individuals with poor CD4 cell recovery before ART onset. Instead, some recent works have been focused on different scenarios, including differential profiles compared to uninfected individuals, the effect of ART among HIV-1-infected individuals, and analysing HIV-1 controllers $^{22,27-29}$. It is important to investigate the dynamic changes in human regulation of miRs during HIV-1 


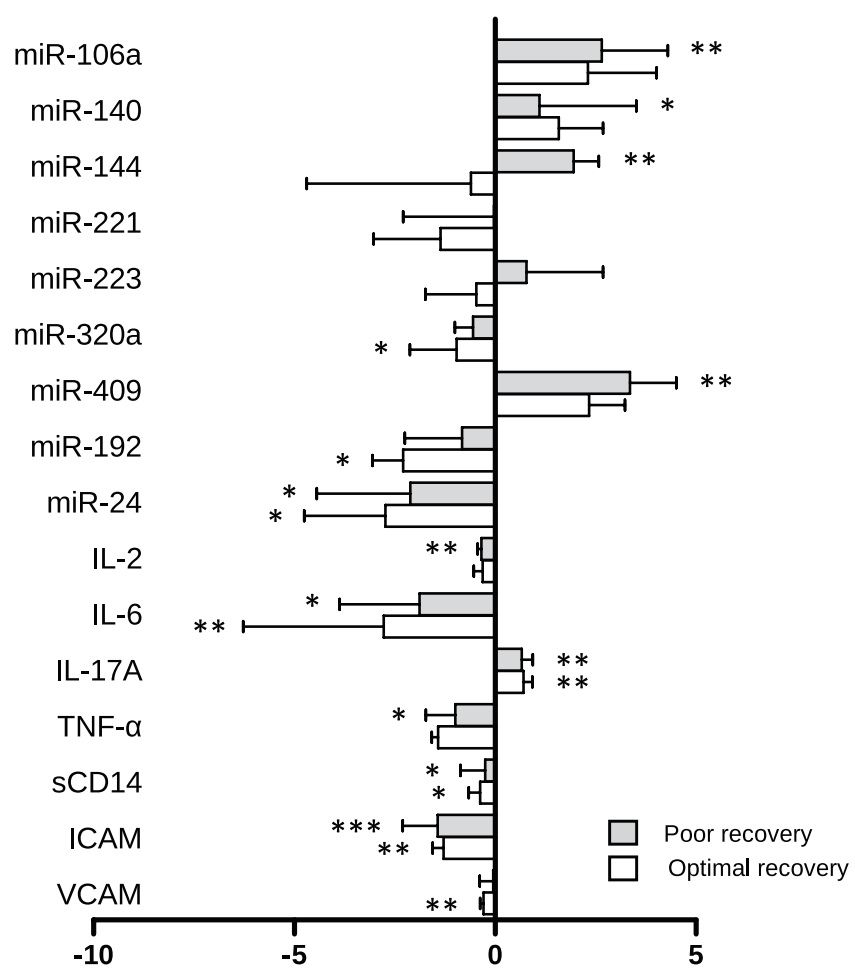

Figure 2. Increments of the levels of miRs and cytokines from ART onset to after 96 weeks of suppressive ART. $* \mathrm{p}<0.05 ; * \mathrm{p}<0.01$ and $* * * \mathrm{p}<0.001$.

infection since they can regulate viral replication and translation and might play an important role in HIV-1 pathogenesis and latency ${ }^{1730,31}$. The interaction of HIV-1 with cellular miRs expressed in infected cells has been controversial. Many groups have reported that cellular miRs bind to HIV and reduce viral gene expression by interfering virion infectivity and virion miR function, facilitating in some degree the HIV-1 latency ${ }^{30-32}$. On the contrary, a few reports have shown that miRNAs bind HIV-1 transcripts very inefficiently ${ }^{19,33}$. However, these last two studies (from the same laboratory) were restricted to early time points after HIV-1 infection, which is a different setting from the present study in which the individuals had a long course of infection.

Although ongoing inflammation (including SCD14 and IL-6) has been previously associated with HIV-1 disease progression, some authors have reported that the baseline sCD14 level was positively associated with CD4 cell recovery ${ }^{34,35}$. These authors suggested a potential explanation where a high level of sCD14 might be protective against LPS-induced immune activation due to a high ratio of SCD14 compared to LPS-binding protein. In contrast, we had previously shown that higher sCD14 level was associated with an increase in markers of HIV-1 progression after suppressive ART ${ }^{36}$, and was found in individuals with low CD4 count after suppressive ART ${ }^{37}$, although no data before ART were available. Besides, we also reported increased levels of IL-6 in individuals with low CD4 cell recovery before ART onset ${ }^{38}$.

In line with our results, miR-192 has been reported to inhibit cell proliferation and induce apoptosis in vitro $^{39,40}$ and rheumatoid arthritis ${ }^{41}$. Also, p53 can induce miR-192 to promote its activation facilitating cell cycle arrest $^{42,43}$. Besides, downregulation of miR-140 has been correlated with higher levels of inflammatory factors in $v_{i t r o}{ }^{44}$ and its upregulation correlated to low levels of IL- 6 among other inflammation cytokines ${ }^{45}$. On the other hand, miR-106a has been reported to prevent Th17-mediated inflammation ${ }^{46,47}$. Of note, this study showed that miR-192 correlated directly with TNF- $\alpha$ and inversely with IL17A, while miR-106a correlated inversely with IL-6 and miR-140 correlated directly with sCD14. On the contrary, miR-106a did not correlate with IL17A in this study perhaps due to the relatively small number of studied individuals. These events are in agreement with our results, where upregulation of miR-192 and, to a lesser extent, downregulation of miR-140 and miR-106a could promote the inhibition of cell proliferation, induction of apoptosis, and increase in inflammatory biomarkers, facilitating the impairment for CD4 recovery in individuals with future poor CD4 cell count recovery under ART.

After 96 weeks of suppressive ART, when individuals were categorised as having either poor CD4 cell recovery or optimal CD4 cell recovery, the expression of miR-106a and miR-140 were still lower in individuals with poor CD4 cell recovery, with a significant increase only in individuals with optimal CD4 cell recovery. Also, the expression of miR-192, although higher compared to individuals with optimal CD4 cell recovery, did not reach statistical significance. Of note, while miR-144 was downregulated compared to individuals with optimal CD4 cell recovery, its levels increased in individuals with optimal CD4 cell recovery and decreased in individuals with poor CD4 cell recovery. This miR has been reported to inhibit inflammatory cytokines like TNF- $\alpha$ and IFN- $\gamma$ during active tuberculosis ${ }^{48}$, which is in line with that found in this study, where the downregulation of miR-144, in combination with the downregulation of miR-106a and miR-140, could facilitate the expression of inflammatory cytokines. Both miR-106a and miR-140 correlated directly with IL-2, which is a multifunctional cytokine 
with potent activity as a $\mathrm{T}$ cell growth factor. Although miR-144 did not correlate with inflammation biomarkers, in contrast to elsewhere reported ${ }^{48}$, miR-192 still correlated inversely with IL-17A, promoting IL17-mediated inflammation.

In parallel, analysing paired samples, the levels of IL-6, and sCD14 decreased during treatment and remained higher in individuals with poor CD4 cell recovery. Besides, the level of IL-2 was lower in individuals with poor CD4 cell recovery, similar to those found at ART onset. After multivariate analysis, only miR-144 has independently associated with CD4 cell recovery with moderate AUC and a slight increase of the probability to be classified to the poor CD4 cell recovery profile.

Limitations of this study included the lack of stored peripheral mononuclear cells to perform cellular immunology analysis, the relatively small number of individuals, and the limited number of paired samples available to analyse the evolution of the biomarkers. Also, there are differences between the groups that could alter the results, such as other viral infections. Besides, the methodology used for this study does not reveal molecular mechanisms about the causes of miRs deregulation. Whether these miRs are actively involved in the progression of HIV-1 infection or are markers for disease pathogenesis remains unclear. On the other hand, the strength of this study is its very restrictive selection criteria that allowed the comparison of two groups of individuals with no overlap in CD4 cell increments that could result in confounding results. Standardised protocols for the quantification of miRs are needed for cross-comparison to enhance their potential as biomarkers. Further studies will be required to determine any mechanistic relationship of miRs to diagnostic characteristics used to identify HIV-1 infected individuals at risk of poor cell recovery after suppressive ART.

Our study provides new evidence on the relationship between miRs and HIV-1 infection that could help improve the management of the individuals at HIV-1 diagnosis (at ART onset). The candidate miRs and cytokines signature sets presented here provide a starting point on which to establish definitive predictive and diagnostic signatures in the important clinical issue of the CD4 cell recovery after antiretroviral treatment. Besides, this signature must also be confirmed in an independent cohort. Also, since exosomes can have different origins, future studies will need to characterise the origin and nature of the HIV-1-associated miRs identified in this study.

\section{Data availability}

The data measured and analysed for the current study are available from the corresponding author upon request.

Received: 15 August 2019; Accepted: 5 February 2020;

Published online: 19 February 2020

\section{References}

1. Rodger, A. J. et al. Mortality in well controlled HIV in the continuous antiretroviral therapy arms of the SMART and ESPRIT trials compared with the general population. AIDS 27, 973-979 (2013).

2. Klatt, N. R., Chomond, N., Douek, D. C. \& Deeks, S. G. Immune activation and HIV persistence: implications for curative approaches to HIV infection. Immunol. Rev. 254, 326-342 (2013).

3. Sobrino-Vegas, P. et al. Impact of late presentation of HIV infection on short-, mid- and long-term mortality and causes of death in a multicenter national cohort: 2004-2013. J. Infect. 72, 587-596 (2016).

4. Pacheco, Y. M. et al. Increased risk of non-AIDS-related events in HIV subjects with persistent low CD4 counts despite cART in the CoRIS cohort. Antiviral Res. 117, 69-74 (2015).

5. Engsig, F. N. et al. Long-term mortality in HIV-positive individuals virally suppressed for $>3$ years with incomplete CD4 recovery. Clin. Infect. Dis. 58, 1312-1321 (2014).

6. Cillo, A. R. et al. Virologic and immunologic effects of adding maraviroc to suppressive antiretroviral therapy in individuals with suboptimal CD4+ T-cell recovery. AIDS 29, 2121-2129 (2015).

7. Negredo, E. et al. Early but limited effects of raltegravir intensification on CD4 T cell reconstitution in HIV-infected individuals with an immunodiscordant response to antiretroviral therapy. J. Antimicrob. Chemother. 68, 2358-2362 (2013).

8. Boer, A. W. et al. A randomized controlled trial evaluating the efficacy and safety of intermittent 3-, 4-, and 5-day cycles of intravenous recombinant human interleukin-2 combined with antiretroviral therapy (ART) versus ART alone in HIV-seropositive patients with 100-300 CD4+ T cells. Clin. Immunol. 106, 188-196 (2003).

9. Massanella, M., Negredo, E., Clotet, B. \& Blanco, J. Immunodiscordant responses to HAART-mechanisms and consequences. Expert Rev. Clin. Immunol. 9, 1135-1149 (2013).

10. Guo, H., Ingolia, N. T., Weissman, J. S. \& Bartel, D. P. Mammalian microRNAs predominantly act to decrease target mRNA levels. Nature 466, 835-840 (2010).

11. Bartel, D. P. MicroRNAs: genomics, biogenesis, mechanism, and function. Cell 116, 281-297 (2004).

12. Bi, Y., Liu, G. \& Yang, R. MicroRNAs: novel regulators during the immune response. J. Cell Physiol. 218, 467-472 (2009).

13. Lodish, H. F., Zhou, B., Liu, G. \& Chen, C. Z. Micromanagement of the immune system by microRNAs. Nat. Rev. Immunol. 8, 120-130 (2008)

14. Swaminathan, G., Navas-Martín, S. \& Martín-García, J. MicroRNAs and HIV-1 infection: antiviral activities and beyond. J. Mol. Biol. 426, 1178-1197 (2014).

15. Narla, V. et al. Unique circulating microRNA profiles in HIV infection. J. Acquir. Immune Defic. Syndr. 79, 644-650 (2018).

16. Duskova, K. et al. MicroRNA regulation and its effect on cellular transcriptome in human immunodeficiency virus 1 (HIV-1) infected individuals with distinct viral load and CD4 cell counts. BMC Infect. Dis. 13, 250 (2013).

17. Squillace, N. et al. Changes in subcutaneous adipose tissue microRNA expression in HIV-infected individuals. J. Antimicrob. Chemother. 69, 3067-3075 (2014).

18. Su, B. et al. Potential application of microRNA profiling to the diagnosis and prognosis of HIV-1 infection. Front. Microbiol. 9,3185 (2018).

19. Bogerd, H. P., Kennedy, E. M., Whisnant, A. W. \& Cullen, B. R. Induced packaging of cellular microRNAs into HIV-1 virions can inhibit infectivity. MBio 8, e02125-16 (2017).

20. Caro-Murillo, A. M. et al. Spanish cohort of naïve HIV-infected individuals (CoRIS): rationale, organization and initial results. Enferm. Infecc. Microbiol. Clin. 25, 23-31 (2007).

21. Garcia-Merino, I. et al. The Spanish HIV BioBank: a model of cooperative HIV research. Retrovirology 6, 27 (2009).

22. Ruiz-de-León, M. J. et al. Lower expression of plasma-derived exosome miR-21 levels in HIV-1 elite controllers with decreasing CD4 T cell count. J. Microbiol. Immunol. Infect. 52, 667-671 (2019). 
23. Livak, K. J. \& Schmittgen, T. D. Analysis of relative gene expression data using real-time quantitative PCR and the 2 (-Delta Delta C(T)) Method. Methods 25, 402-408 (2001).

24. Mestdagh, P. et al. A novel and universal method for microRNA RT-qPCR data normalization. Genome Biol. 10, R64 (2009).

25. Farina, N. H. et al. Standardizing analysis of circulating microRNA: clinical and biological relevance. J. Cell Biochem. 115, 805-811 (2014).

26. Chehimi, J. et al. Baseline viral load and immune activation determine the extent of reconstitution of innate immune effectors in HIV-1-infected subjects undergoing antiretroviral treatment. J. Immunol. 179, 2642-2650 (2017).

27. Liu, M. Q. et al. Antiretroviral therapy fails to restore levels of HIV-1 restriction miRNAs in PBMCs of HIV-1-infected MSM. Medicine (Baltimore) 94, e2116 (2015)

28. Egaña-Gorroño, L. et al. Differential microRNA expression profile between stimulated PBMCs from HIV-1 infected elite controllers and viremic progressors. PLoS One 9, e106360 (2014).

29. Reynoso, R. et al. MicroRNAs differentially present in the plasma of HIV elite controllers reduce HIV infection in vitro. Sci. Rep. 4, 5915 (2014).

30. Chen, B. S. \& Li, C. W. Constructing an integrated genetic and epigenetic cellular network for whole cellular mechanism using highthroughput next-generation sequencing data. BMC Syst. Biol. 10, 18 (2016).

31. Lu, X. et al. High-throughput sequencing identifies HIV-1-replication- and latency-related miRNAs in CD4 ${ }^{+} \mathrm{T}$ cell lines. Arch. Virol. 162, 1933-1942 (2017).

32. Chang, S. T. et al. Next-generation sequencing of small RNAs from HIV-infected cells identifies phased microRNA expression patterns and candidate novel microRNAs differentially expressed upon infection. mBio 4, e00549-12 (2013).

33. Whisnant, A. W. et al. In-depth analysis of the interaction of HIV-1 with cellular microRNA biogenesis and effector mechanisms. mBio 4, e00193-13 (2013).

34. Shivakoti, R. et al. Effect of baseline micronutrient and inflammation status on CD4 recovery post-cART initiation in the multinational PEARLS trial. Clin. Nutr. 38, 1303-1309 (2019).

35. Rajasuriar, R. et al. Biological determinants of immune reconstitution in HIV-infected individuals receiving antiretroviral therapy: the role of interleukin 7 and interleukin 7 receptor $\alpha$ and microbial translocation. J. Infect. Dis. 202, 1254-1264 (2010).

36. Sandler, N. G. et al. Plasma levels of soluble CD14 independently predict mortality in HIV infection. J. Infect. Dis. 203, 780-790 (2011).

37. Romero-Sánchez, M. et al. Different biological significance of sCD14 and LPS in HIV-infection: importance of the immunovirology stage and association with HIV-disease progression markers. J. Infect. 65, 431-438 (2012).

38. Rosado-Sánchez, I. et al. Higher levels of IL-6, CD4 turnover and Treg frequency are already present before cART in HIV-infected subjects with later low CD4 recovery. Antiviral Res. 142, 76-82 (2017).

39. Ke, S. et al. MicroRNA-192 regulates cell proliferation and cell cycle transition in acute myeloid leukemia via interaction with CCNT2. Int. J. Hematol. 106, 258-265 (2017).

40. Wang, Y. et al. Upregulation of miR-192 inhibits cell growth and invasion and induces cell apoptosis by targeting TCF7 in human osteosarcoma. Tumour Biol. 37, 15211-15220 (2016).

41. Li, S., Jin, Z. \& Lu, X. MicroRNA-192 suppresses cell proliferation and induces apoptosis in human rheumatoid arthritis fibroblastlike synoviocytes by downregulating caveolin 1. Mol. Cell Biochem. 432, 123-130 (2017).

42. Sun, C. Y., Zhang, X. P. \& Wang, W. Coordination of miR-192 and miR-22 in p53-mediated cell fate decision. Int. J. Mol. Sci. 20, 4768 (2019).

43. Song, B. et al. miR-192 Regulates dihydrofolate reductase and cellular proliferation through the p53-microRNA circuit. Clin. Cancer Res. 14, 8080-8086 (2008).

44. Yang, Y. et al. Upregulation of miRNA-140-5p inhibits inflammatory cytokines in acute lung injury through the MyD88/NF-kB signaling pathway by targeting TLR4. Exp. Ther. Med. 16, 3913-3920 (2018).

45. Zhang, Q. et al. Overexpression of miR-140-5p inhibits lipopolysaccharide-induced human intervertebral disc inflammation and degeneration by downregulating toll-like receptor 4. Oncol. Rep. 40, 793-802 (2018).

46. Kästle, M. et al. microRNA cluster 106a 363 is involved in T helper 17 cell differentiation. Immunology 152, 402-413 (2017).

47. Yang, J. et al. MicroRNA-106a provides negative feedback regulation in lipopolysaccharide-induced inflammation by targeting TLR4. Int. J. Biol. Sci. 5, 2308-2319 (2019).

48. Liu, Y. et al. Modulation of T cell cytokine production by miR-144* with elevated expression in patients with pulmonary tuberculosis. Mol. Immunol. 48, 1084-1090 (2011).

\section{Acknowledgements}

This work was supported in part by the Spanish AIDS Network "Red de Investigación en Sida (RIS)" RD16/0025/0001; Ayudas contratación de ayudantes de investigación y técnicos de laboratorio, Consejería de Educación, Juventud y Deporte de la Comunidad de Madrid (PEJ15/bio/tl-0064 to MJRL and PEJ17/AI_BMD6683 to FHW); Health Institute Carlos III, PI18/01216; Junta de Andalucía, Consejería de Economía, Innovación, Ciencia y Empleo, Proyecto de Investigación de Excelencia CTS2593; YMP was supported by the Consejería de Salud y Bienestar Social of Junta de Andalucía through the "Nicolás Monardes" programme (C-0013-2017).

\section{Author contributions}

A.V. and Y.M.P. designed the experiments; A.V., F.H.W., M.J.R.L., I.R.S. and E.V. performed the experiments; A.V. and Y.M.P. wrote the manuscript; M.L., S.M., F.V. and J.B. reviewed and contributed to the discussion. All authors revised the manuscript and gave final approval of the current version.

\section{Competing interests}

The authors declare no competing interests.

\section{Additional information}

Supplementary information is available for this paper at https://doi.org/10.1038/s41598-020-60073-8.

Correspondence and requests for materials should be addressed to Y.M.P. or A.V.

Reprints and permissions information is available at www.nature.com/reprints.

Publisher's note Springer Nature remains neutral with regard to jurisdictional claims in published maps and institutional affiliations. 
(c) (i) Open Access This article is licensed under a Creative Commons Attribution 4.0 International License, which permits use, sharing, adaptation, distribution and reproduction in any medium or format, as long as you give appropriate credit to the original author(s) and the source, provide a link to the Creative Commons license, and indicate if changes were made. The images or other third party material in this article are included in the article's Creative Commons license, unless indicated otherwise in a credit line to the material. If material is not included in the article's Creative Commons license and your intended use is not permitted by statutory regulation or exceeds the permitted use, you will need to obtain permission directly from the copyright holder. To view a copy of this license, visit http://creativecommons.org/licenses/by/4.0/.

(C) The Author(s) 2020 